\title{
The Importance of the Owner / Manager's Profile in Accessing Banking Credit for SMES in the Trade / Distribution Branch in the Context Of Information Asymmetry
}

(L'importance Du Profil Du Proprietaire/Dirigeant Dans L'acces Au Credit Bancaire Des Pme De La Branche Commerce/Distribution En Contexte D'asymetrie D'information)

\section{DJOU André Ilaire, KUIPOU TOUKAM Christophe, TAKOUDJOU NIMPA Alain,}

$\mathrm{PhD}$ student in management sciences at the Faculty of Economics and Management of the University of Dschang-Cameroon.

Assistant at the National Higher School of Technical Education (ENSET) in Bambili, University of Bamenda-Cameroon.

Lecturers, Associate of Management Sciences, Faculty of Economic Sciences and Management of the University of Dschang-Cameroon.

\begin{abstract}
The purpose of this article is to examine the impact of the various dimensions of the owner / manager profile on access to bank credit for Cameroonian SMEs working in the trade / distribution subsector. The results of the estimates carried out on a convenience sample of 93 SMEs / CDs highlight three main explanatory variables for access to bank credit, including the age of the owner / manager, his level of education and his relationship network intensity. Thus, owners / managers of SMEs/CDs aged 40 and over, with a high school education and a high network intensity are more likely to access the amount of credit requested. Therefore, promoters of SMEs / CDs would be better off establishing links with bankers, suppliers of goods or even associations in order to increase their chance of accessing the amount of credit requested.
\end{abstract}

Keywords: access to credit, SME in the trade / distribution subsector, logistic regression, relational network, manager

\section{Introduction}

La problématique du financement des entreprises est ancienne. Elle remonte aux travaux pionniers de Modigliani et Miller (1958) et demeure d'actualité en dépit du nombre important de travaux déjà effectués sur le sujet aussi bien dans les pays développés (Ross, 1977 ; Myers et Majluf, 1984 ; Fathi et Gally, 2003 ; Colot et Croquet, 2007a) que dans les pays en voie de développement (Aydi, 2003 ; Ngongang, 2018). Cette problématique est d'autant plus actuelle car plusieurs chefs d'entreprises continuent à évoquer le problème d'accès au crédit bancaire comme obstacle à leur croissance. Ce problème se pose également dans les $\mathrm{PME}^{1}$ caractérisées par l'asymétrie d'information et une production d'information financière incomplète et généralement peu fiable (Ndjanyou, 2001). Cette situation n'épargne pas les entreprises camerounaises.

\footnotetext{
${ }^{1}$ La définition officielle de PME au Cameroun découle de la loi n ${ }^{\circ}$ 2015/010 du 16 juillet 2015 modifiant et complétant la loi ${ }^{\circ}$ 2010/001 du 13 avril 2010 portant promotion des Petites et Moyennes Entreprises (PME). Selon cette loi, rentrent dans la catégorie PME les entreprises ayant un effectif permanent inférieur ou égale à 100 individus et dont le chiffre d'affaires annuel hors taxe est inférieur ou égale à trois milliard de FCFA.
} 
Ainsi, dans un contexte comme celui du Cameroun où plusieurs travaux ont déjà été effectués sur le financement bancaire des PME (notamment des travaux de Wamba et Tchamanbé-Djiné, 2002 ; Wanda, 2007 ; Ngantchou, 2010 ; Feudjo et Tchankam, 2012 ; Djoutsa et al., 2018) et où l'Etat n'a cessé de créer des structures d'appui et soutien à ces entreprises pour résoudre ce problème (création du Fonds de Garantie et d'Aide aux Petites Entreprises, du Programme Intégré d'Appui aux Acteurs du Secteur Informel, du Programme d'Appui à la Jeunesse Rurale et Urbaine ou récemment de la Banque Camerounaise des PME, de l'Agence de Promotion des PME, des Centres de Gestion Agréés ou encore des Centres de Formalités de Création d'Entreprises), plusieurs promoteurs de PME ne parviennent pas toujours à accéder convenablement aux ressources financières dont ils ont besoin pour le financement de leurs cycles d'exploitation et de financement. À ce titre, 30,7\% des promoteurs d'entreprises affirment que l'accès au crédit bancaire demeure un frein à l'entreprenariat au Cameroun d'après l'INS (2018). Il ressort également du rapport de l'INS (2018) que huit (08) entreprises sur dix (10) financent leurs activités par fonds propres au Cameroun.

Ce problème se pose avec plus d'acuité dans les PME relevant de la branche commerce/distribution (PME/CD) au regard de leur nombre à l'échiquier national (les entreprises relevant de ce sous-secteur sont majoritaires aussi bien sur le plan global (soit 51,39\% de l'ensemble des entreprises en 2016 d'après l'INS) que sur le plan sectoriel (soit 61,1\% des entreprises du secteur tertiaire en 2016 d'après l'INS)). Les principales caractéristiques de ces entreprises sont la personnalisation de leur mode de fonctionnement, l'asymétrie d'information et les pratiques et système de gestion parfois rudimentaires qui ne satisfont pas toujours les exigences de la banque (Schwarz, 2011). La forte centralisation de la gestion apparaît également comme l'un des critères essentiels de définition de cette catégorie de PME.

Cependant, si de nombreuses études (Jensen et Meckling, 1976 ; Myers et Majluf, 1984 ; Colot et Croquet, 2007) ont mis en exergue plusieurs facteurs explicatifs du niveau d'endettement bancaire des entreprises, rares sont celles qui se sont intéressées au profil du dirigeant pour expliquer ce dernier. Ainsi, de nombreux auteurs militent pour la très forte influence du dirigeant de la PME sur son système de gestion (Julien, 1990 ; Coupal, 1994). À ce sujet, D'ambroise (1997) affirme : "l'entreprise c'est lui, l'argent de l'entreprise, c'est le sien, les décisions sont centralisées, le propriétaire/dirigeant est le preneur de risque, le stratège ». Allant dans le même sens, Cooper (1993) pense que la personnalité de l'entrepreneur fait partie des facteurs essentiels à la réussite de l'entreprise et à la conquête du banquier. McClelland (1961), Gartner (1988), Saporta (1994), Berger et Udell (1988) montrent qu'il est intéressant pour les banquiers de tenir compte au cours de leur décision d'octroi de crédit des éléments qui touchent le propriétaire-dirigeant de l'entreprise.

Dès lors, la PME/CD peut être définie comme un système social qui tourne autour de l'entrepreneur (Filion, 1991). Ce dernier assume quasi-exclusivement les responsabilités administratives, financières et sociales et participe activement à la gestion courante de son entreprise. Plusieurs études (Berger et Udell, 1988 ; Colot et Croquet, 2007 ; Djoutsa et al., 2018) ont identifié et mis en exergue des éléments caractéristiques du profil $^{2}$ du dirigeant tels que l'âge, le niveau d'étude et son réseau relationnel et leur impact sur l'accès au crédit bancaire.

Parmi ces éléments, l'âge managérial renvoie au nombre d'années passées par le propriétaire/dirigeant à la tête de l'entreprise depuis la création) et le niveau d'étude désigne quant à lui, le savoir ou expérience ayant un lien avec les activités pratiquées dans la PME. La notion de réseau relationnel constitue quant à elle, le capital social du promoteur. Ce concept trouve ses origines dans la description des ressources relationnelles encastrées dans les réseaux sociaux (Nkakleu, 2003) et met en évidence d'après Aydi (2003), trois dimensions qui peuvent permettre d'en mesurer l'intensité : la taille du réseau relationnel, la nature et la

\footnotetext{
${ }^{2}$ Le profil peut être défini comme : "un ensemble des principaux traits distinctifs d'une personne, d'un groupe ou d'une chose. C'est aussi l'ensemble des qualifications, des aptitudes et des traits psychologiques d'une personne » (Boukar et Tsapi, 2011)
} 
qualité des liens sociaux. En général, les bailleurs de fonds n'accordent de crédit qu'aux personnes en qui ils ont un minimum de confiance ou envers lesquelles ils ont un devoir psychologique ou moral. Cela suppose l'entretien de liens étroits avec des personnalités politiques haut placées, des cadres de l'administration ou encore des dirigeants des institutions financières. L'existence d'affinités ou l'entretien de liens de fraternité avec des fournisseurs ou des clients, l'appartenance à des groupes associatifs culturels ou d'affaires sont susceptibles de conférer un avantage certain dans la course aux financements (Aydi, 2003). Uzzi (1999) affirme à ce sujet que les liens sociaux facilitent le transfert des informations entre prêteurs et emprunteurs.

De plus, la théorie du capital social telle que développée dans le champ de la sociologie économique par des auteurs comme Granovetter (1973), Bourdieu (1980), Coleman (1990), Putnam (1995), met en exergue la capacité qu'ont les individus de tirer des bénéfices et des ressources des réseaux sociaux auxquels ils appartiennent. Dans les relations financières, le capital social permet d'améliorer l'information sur le risque de l'emprunteur (Baudasse et Montalieu, 2005). D'ailleurs, Aydi (2003) affirme qu'il n'est pas nouveau de dire que les relations sociales entre le banquier et l'entrepreneur ont des effets positifs sur les conditions de crédit. Plus ce lien sera fort plus les chances d'obtenir des crédits seront grandes. Cependant, il est souvent reproché au capital social son fort impact qui peut contribuer à ne pas justifier un degré élevé de formalisation de l'information et pas simplement sur le plan financier; son usage conduit souvent à une vision et à des prises de décision à court terme ; la concentration du pouvoir autour du dirigeant se traduit par le développement de relations en tête à tête qui ont pour conséquence de limiter les choix de l'entreprise. En résumé, dans un contexte comme celui du Cameroun où de nombreuses études se sont penchées sur les facteurs explicatifs du niveau d'endettement bancaire des entreprises sans toutefois mettre l'accent sur les $\mathrm{PME} / \mathrm{CD}$, où la majorité des entreprises sont des entreprises individuelles, où il y a un manque de consensus sur le signe de certaines variables (par exemple, les travaux de Berger et Udell (1988) ainsi que ceux de Djoutsa et al. (2018) aboutissent à la conclusion selon laquelle l'âge du promoteur de l'entreprise influence positivement l'accès au crédit bancaire tandis que ceux de Colot et Croquet (2007a) aboutissent plutôt au contraire), nous nous demandons quels sont les éléments du profil du propriétaire/dirigeant des PME de la branche commerce/distribution qui leur permettent d'accéder au crédit bancaire. Autrement dit, Quel est l'impact du profil du propriétaire/dirigeant sur l'accès au crédit des PME du sous-secteur commerce/distribution au Cameroun?

Dans cette perspective, l'objet de cet article est d'analyser l'influence du profil du propriétaire/dirigeant sur l'accès au crédit bancaire des PME/CD. Pour y parvenir, cet article est organisé autour de trois points. Le premier point, de nature théorique, permet d'exploser les théories et travaux empiriques qui existent dans la littérature sur l'accès au crédit des entreprises de petites dimensions et la formulation des hypothèses. Le deuxième point expose la démarche méthodologique adoptée en vue de tester les hypothèses formulées. Les résultats issus des analyses statistiques feront l'objet d'une discussion dans un troisième point. Une formulation des implications managériales, puis une présentation des limites et des voies de recherche seront enfin envisagées.

\section{I- Fondements Théoriques De L'étude Et Hypothèses De Recherche}

Le problème du financement du besoin d'investissement et du besoin en fonds de roulement des entreprises est au cœur de la problématique de la structure financière optimale. A la question de savoir « faut-il se financer par fonds propres ou par endettement bancaire », les avis sont partagés. D'aucuns, à l'instar de Modigliani et Miller (1958) pensent que les formes d'endettement (l'endettement bancaire ou l'emprunt obligataire) sont neutres sur la valeur de l'entreprise tandis que d'autres estiment que l'endettement constitue un signal de la bonne santé financière de l'entreprise. Pour ces derniers, il se pose le problème d'accès au financement bancaire surtout en ce qui concerne les PME à cause de l'asymétrie d'information qui entoure la relation de crédit. Dès lors, plusieurs théories et travaux empiriques existent dans la littérature et tentent 
d'expliquer l'accès au crédit bancaire des entreprises de petite dimension. Dans ce qui suit, il est présenté d'une part, les théories qui expliquent l'accès des PME au crédit et d'autre part, une revue empirique de la littérature qui conduit à la déduction des hypothèses.

\section{1- Théories explicatives de l'accès au crédit bancaire des entreprises}

Belletante, Levratto et Paranque $(2001)^{3}$ déclarent : «...les faits ne peuvent pas être considérés comme indépendants des théories qui les étudient». Dès lors, il est admis d'étudier les déterminants de l'accès au crédit des PME à la lumière des théories financières traditionnelles. En effet, les PME seraient soumises aux mêmes problèmes fondamentaux évoqués par la théorie financière notamment les problèmes d'agence et d'asymétries informationnelles. De plus, " il n'y a aucune théorie universelle expliquant les choix d'endettement et aucune raison de s'attendre à une. Il existe toutefois plusieurs théories conditionnelles qui peuvent être utiles » (Stewart et Myers, 2001). Nombreuses sont donc les théories qui mettent en exergue les facteurs explicatifs de l'accès des PME aux sources de financement formel.

\subsection{1- L'apport de la théorie de l'agence}

Le contrat existant entre créancier et débiteur constitue le second niveau d'analyse de la relation d'agence telle que présentée dans les travaux pionniers de Jensen et Meckling (1976). S'inscrivant à la suite de ces travaux, nombreux sont les auteurs qui pensent que la théorie de l'agence développée en contexte de grandes entreprises est également pertinente dans l'explication des problématiques des PME (Ang, 1992 ; Belletante et Levratto, 1995). Ainsi, partant du principe que l'entreprise est un nœud de contrats entre différents acteurs dont chacun cherche la maximisation de son propre intérêt, cette théorie a été développée à partir des relations entre actionnaires propriétaires de l'entreprise en tant que principal d'une part, et les dirigeants en tant qu'agents d'autre part. Appliquée aux PME, la théorie de l'agence décrit les conditions selon lesquelles le propriétaire-dirigeant fait office d'agent pour les bailleurs de fonds (Jensen et Meckling, 1976).

En somme, l'endettement permet une réactualisation des hypothèses de la théorie de l'agence en contexte PME et notamment dans un contexte où l'actionnaire joue le rôle de propriétaire/dirigeant. Le postulat de rationalité sur lequel se fonde la théorie de l'agence rend plausible les hypothèses formulées par Watts et Zimmerman (1990) : le propriétaire-dirigeant peut à priori instrumentaliser les données comptables afin de présenter une image positive de son entreprise ; à posteriori, les données comptables peuvent également être instrumentalisées dans le but d'éviter la violation de certains seuils contractuels.

\subsection{2- Théorie de la contingence et déterminants de l'accès au crédit bancaire}

La contingence est un concept clé en matière d'analyse des organisations et se définit comme une situation spécifique et évolutive qui conduit à rejeter les prescriptions uniques et standards. L'école de la contingence s'inscrit dans le prolongement des travaux de l'école sociologique. Formulée en 1958 à partir des travaux de plusieurs auteurs tels que Lawrence et Lorch (1967), elle abandonne définitivement le préalable, longtemps dominant du « one best way » des écoles classiques et des relations humaines et tente de rendre compte de la manière dont se structurent les organisations en étudiant l'influence des variables de contexte sur les caractéristiques des organisations. Cette théorie reconnait que toutes les entreprises sont différentes et qu'il $n$ y a pas de structure formelle qui soit idéale. À l'image de la théorie financière classique, de la théorie des droits de propriété ou encore de la théorie de l'agence, elle met en relief les déterminants du comportement financier des entreprises.

$\mathrm{Au}$ regard de ces postulats de base (inexistence d'une structure organisationnelle qui soit la meilleure, structure organisationnelle comme résultat d'une double dynamique de différentiation et d'intégration, adaptation des entreprises à leur environnement) nous constatons que les facteurs environnementaux,

${ }^{3}$ In Diversité économique et modes de financement des PME. Une présentation de l'ouvrage paru chez L'Harmattan collection « Economiques » Humanisme et Entreprise - numéro 249 octobre 2001. 
culturels, structurels et les décisions propres à l'entreprise influencent fortement l'accès effectif au crédit bancaire. D'après cette théorie, l'on ne peut ignorer une approche contingente du fait que les PME sont hétérogènes mais, sans oublier leurs similitudes communes. En effet, nous pensons par exemple que les caractéristiques du propriétaire/dirigeant de la PME sont susceptibles d'influencer la probabilité d'accès au crédit bancaire.

\subsection{3- L'apport de la théorie du capital social}

Cette théorie, comme développée en sociologie économique par des auteurs à l'instar de Granovetter (1973), Bourdieu (1980) ou encore Coleman (1990), souligne la capacité qu'ont les personnes physiques et morales d'extraire des bénéfices et des ressources de leur réseau relationnel. Elle a comme postulat de base, le fait que les réseaux de relations sociales constituent une ressource précieuse pour la conduite des affaires car ils facilitent l'action économique (Nahapiet et Ghoshal, 1998) et permettent aux entrepreneurs d'élargir leur champ d'action, d'économiser leurs moyens et d'accéder à des ressources et opportunités exclusives.

Les recherches récentes sur la théorie du capital social, notamment en finance, mettent en évidence l'importance du capital social dans la facilitation de l'accès au crédit bancaire (Uzzi, 1999). Le concept de capital social a connu son affinement avec les travaux de Bourdieu, Boltanski et De Saint-Martin (1973). Relancé à la fin des années quatre-vingt par Coleman (1990) et au cœur des débats politiques dans les années 90 sous l'impulsion de Putnam (1995), le capital social, concept devenu usuel, est désormais perçu comme un ensemble de relations sociales mutuellement avantageuses. Il facilite les actions au sein d'un groupe (association, réseau, etc.) en passant par la coordination et la coopération et est entretenu par la confiance (Coleman, 1990). En effet, la survie d'une entreprise dépend de sa capacité à se créer des opportunités et à développer de nouveaux partenariats. Dans ce sens, le capital social, sous la forme de la confiance entre acteurs facilite les échanges, la création et le développement de partenariats stratégiques. Le capital social peut donc être considéré ici comme une forme de garantie car peut faciliter l'accès au crédit bancaire des PME. À ces différentes théories, se sont ajoutées des investigations empiriques.

\section{2- Formulations des hypothèses}

S'inscrivant dans le courant de pensée de la finance comportementale, l'idée maîtresse à la base de cette étude est celle selon laquelle l'accès au crédit bancaire est fortement influencé par le profil du dirigeant de l'entreprise en dehors des caractéristiques de l'entreprise et des facteurs environnementaux. Dans cette mouvance, l'étude menée par Chebil et Levy-Tadjiné (2010) auprès de 65 chargés de clientèle des principales banques tunisiennes montrent que les banquiers sont très sensibles au profil du propriétaire/dirigeant de la PME qui, selon eux, est un élément déterminant dans la réussite de l'entreprise et par ricochet dans la capacité de l'entreprise à rembourser ses dettes. Boukar et Tsapi (2011) définissent le profil comme un ensemble des principaux traits distinctifs d'une personne, d'un groupe ou d'une chose ; c'est aussi l'ensemble des qualifications, des aptitudes et des traits psychologiques d'une personne. Ces traits peuvent être l'âge, le réseau relationnel, la formation reçue (niveau et type), le caractère conservateur ou encore les relations interpersonnelles.

L'entreprise peut être définie comme un système social qui tourne autour de l'entrepreneur (Filion, 1991). Ce dernier assure quasi-exclusivement les responsabilités administratives, financières et sociales et participe activement à la gestion courante de son entreprise. C'est donc à juste titre qu'on peut penser que le profil de ce dernier peut influencer positivement la probabilité d'accès de l'entreprise au crédit bancaire. En effet, Cooper (1993) estime que la personnalité de l'entrepreneur fait partie des facteurs essentiels à la réussite de l'entreprise et à «la conquête du banquier».

L' « effet d'Egotrophie ${ }^{4} »$ caractérise généralement le propriétaire/dirigeant des PME. A ce sujet, D'Amboise (1997) affirme: "L'entreprise c'est lui ; l'argent de l'entreprise, c'est le sien, les décisions sont

\footnotetext{
${ }^{4}$ Phénomène qui traduit la prépondérance du rôle du propriétaire/dirigeant dans les PME et qui conduit parfois ce dernier à éprouver le sentiment d'être indispensable jusqu'à l'excès. Inclinaison d'un dirigeant de PME à tout ramener à sa propre personne.
} 
centralisées, le propriétaire/dirigeant est le premier preneur de risque, le stratège». D’après Cooper (1993), la personnalité de l'entrepreneur fait partie des facteurs essentiels à la réussite de l'entreprise et à "la conquête du banquier ».

Ainsi, la littérature sur la gestion stratégique et sur les ressources humaines nous permet de distinguer trois types de profil de dirigeant : le profil biographique (genre et âge notamment), le profil des compétences managériales (niveau scolaire, type de formation reçue par exemple) et le pouvoir de prestige et d'expertise du dirigeant.

En ce qui concerne l'âge du propriétaire-dirigeant, ce critère est très important pour les banques car le fondateur de l'entreprise est souvent lui-même le dirigeant et s'il est trop jeune, il peut manquer d'expérience dans les affaires. Woywode et Lessat (2001) trouvent que les jeunes propriétaires-dirigeants sont plus motivés par une forte croissance et par l'innovation, contrairement aux plus âgés. L'étude menée par Kabacoff et Stoffey (2001) sur les différences multi générationnelles de comportement en tant que leader et leur performance individuelle montrent que les jeunes propriétaires-dirigeants dont l'âge oscille entre 25 et 35 ans travaillant avec beaucoup d'énergie et d'intensité de manière à atteindre des résultats de haut niveau, ont tendance à prendre des risques. Les dirigeants âgés quant à eux, sont ceux qui ont une bonne performance et sont capables d'emmener l'entreprise à générer des ressources. Par conséquent, l'âge apparait donc comme un élément sur lequel on peut se baser pour expliquer les comportements et les attitudes des individus. Les entretiens menés par Chebil et Levy Tadjine (2010) auprès de banques tunisiennes et ceux menés par Um-Ngouem et Ekonne (2013) auprès des IMF au Cameroun, indiquent que l'âge du dirigeant est l'un des éléments pris en considération au même titre que le marché de l'entreprise et les aspects financiers du projet (le budget prévisionnel ou encore le plan de financement). D'après l'étude d'Um-Ngouem et Ekonne (2013) sur un échantillon d'IMF au Cameroun, il s'avère que plus de 50,3\% des chargés de la clientèle retiennent ce critère dans leur décision d'octroi de crédit. Ainsi, plus l'entrepreneur est âgé, plus il a une forte propension à rembourser ses crédits. Suite à ces travaux, nous pouvons déduire l'hypothèse suivante :

$H_{1}$ : L'âge du propriétaire/dirigeant de la PME/CD influence positivement l'accès au montant du crédit bancaire sollicité.

Concernant le niveau scolaire et le type de formation reçue, des auteurs à l'image de Janssen et Wtterwulghe (1998) pensent que ces variables ont une influence sur la réussite de l'entreprise. Les travaux de Woywode et Lessat (2001) démontrent par exemple qu'une formation universitaire de qualité a une conséquence sur la croissance de l'entreprise. Trulsson (1999) affirme quant à lui, à travers des recherches effectuées en Tanzanie, en Ouganda et au Zimbabwe, que les PME les plus productives et compétitives sont celles dirigées par des entrepreneurs ayant au moins le baccalauréat. Dès lors, la formation aide le propriétaire/dirigeant de l'entreprise à éviter de prendre de mauvaises décisions et lui donne une prédisposition à mieux agir que celui qui n'en a pas. En toute logique, on peut donc penser que ces variables puissent retenir l'attention des banques lors de leur décision d'octroi de crédit. Suite aux conclusions de ces études, nous formulons l'hypothèse suivante :

$\boldsymbol{H}_{2}$ : Moins le niveau d'étude du propriétaire/dirigeant de la PME/CD est élevé, faible est sa probabilité d'accéder au montant du crédit bancaire sollicité.

Le dirigeant ayant une formation en gestion est capable d'analyser et d'interpréter les états financiers, ce qui, d'après les banquiers, diminue le risque financier. Selon lui, le niveau d'endettement bancaire des PME dirigées par des gestionnaires est plus élevé que celui des PME dirigées par les non gestionnaires. De plus, St-Pierre (2004) indique que la connaissance du dirigeant de la PME est une source de performance. Selon l'auteur le dirigeant ayant une bonne connaissance en gestion est capable d'opérer des contrôles financiers réduisant ainsi le risque de faillite de l'entreprise. Cette connaissance en gestion peut donc séduire le 
banquier. De plus, connaissant la vertu du financement bancaire avec notamment les économies d'impôts qu'il procure, le dirigeant gestionnaire serait plus incité à recourir davantage à l'endettement bancaire. Nous déduisons l'hypothèse suivante :

$\boldsymbol{H}_{3}$ : Le fait pour le propriétaire/dirigeant de la PME/CD d'avoir suivi une formation particulière en sciences de gestion

augmente significativement sa probabilité d'accéder au montant du crédit sollicité.

Dans la littérature récente sur l'entreprenariat, le capital social est évoqué comme le facteur apte à réduire les coûts de transaction des banques dans la surveillance de leurs clients et partant, devrait atténuer les effets pervers de l'asymétrie d'information. Aydi (2003), trouvait déjà à travers une étude menée auprès de 31 firmes tunisiennes qu'avoir un réseau relationnel plus large, entretenir des relations interpersonnelles plus étroites avec son banquier et entretenir davantage des liens avec des personnes haut placées impactent positivement sur l'accès au crédit bancaire. Feudjo et Tchankam (2012), à travers une étude menée sur un échantillon de 62 Petites et Moyennes Industries (PMI) camerounaises affirment que les banques n'accordent en général de crédits qu'aux entreprises en qui elles ont un minimum de confiance ou en vers lesquelles elles ont un devoir psychologique ou moral. Ainsi, l'existence d'affinités, l'appartenance à des groupes associatifs culturels, traditionnels ou d'affaires sont susceptibles de conférer un avantage certain dans l'accès au crédit des entreprises. Les résultats de ces travaux nous permettent de formuler l'hypothèse suivante :

$H_{4}$ : Le réseau relationnel du propriétaire/dirigeant de la PME/CD impacte positivement sur la probabilité d'accéder au montant du crédit sollicité.

La figure 1 présente schématiquement le cadre conceptuel qui sied à cette étude.

\section{Figure 1 : Cadre conceptuel de l'étude}

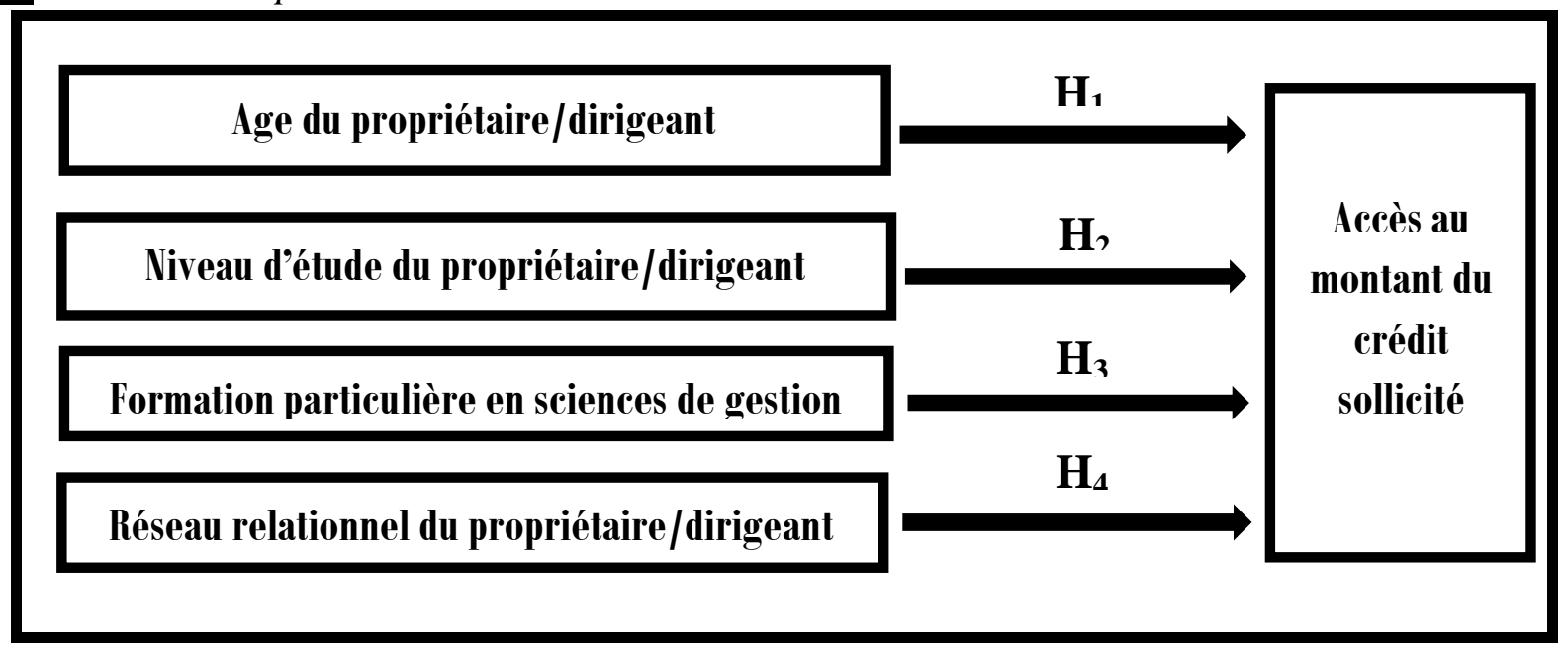

Source : auteur à partir d'une revue exhaustive de la litterature

$\mathrm{Au}$ regard des développements présentés ci-dessus et du cadre conceptuel sous-jacent, on suppose qu'il existe une relation entre le profil du propriétaire/dirigeant de la PME du sous-secteur commerce/distribution et l'accès au crédit bancaire ; bien qu'il soit difficile d'arriver à un consensus sur le sens de la relation pour les différentes dimensions. Notre étude empirique sur un échantillon de PME camerounaises ayant sollicité un crédit bancaire vise, dans les rubriques suivantes, à vérifier ce cadre conceptuel.

\section{II- MÉTHODOLOGIE DE LA RECHERCHE}

Toutes les études et analyses économétriques nécessitent une méthode de recherche. Dans cette mouvance, il est présenté dans ce qui suit la procédure d'échantillonnage, la mesure des variables et les caractéristiques des entreprises de l'échantillon.

\section{1- Choix de l'échantillon et collecte des données}


Les données sur l'importance que revêt le profil du propriétaire/dirigeant de la PME/CD dans l'accès au crédit bancaire sont de source primaire. Elles ont été

collectées par un questionnaire ${ }^{5}$ administré en face à face aux propriétaires/dirigeants des PME/CD de l'échantillon. Ce questionnaire est organisé en deux rubriques : la première s'intéresse aux caractéristiques de l'entreprise, la seconde se focalise sur le profil du propriétaire/dirigeant de la PME. Seules les PME/CD ayant déjà bénéficié du crédit bancaire par le passé ont été retenues. Grâce à la méthode de convenance, un échantillon de 93 PME/CD a été finalement constitué.

\section{2- Modèle économétrique de l'étude et opérationnalisation des variables}

En considérant que le profil du propriétaire/dirigeant de la PME du sous-secteur commerce/distribution peut avoir une influence sur l'accès au crédit bancaire et en s'appuyant sur les travaux de Djoutsa et al. (2018), nous avons construit le modèle économétrique suivant (où la probabilité d'accéder au montant du crédit sollicité est représentée par la variable binaire $C R E D R E C U)$ :

$\boldsymbol{C R E D R E C U}_{i}=v_{0}+v_{1} \boldsymbol{A P}_{i}+v_{2} \boldsymbol{N E P}_{i}+v_{3} \boldsymbol{F P S G}_{i}+v_{4} \boldsymbol{I R E S P}_{i}+\boldsymbol{\varepsilon}_{i}$

Le tableau 1 ci-après présente une synthèse de l'opérationnalisation des variables de ce modèle.

Tableau 1 : Synthèse des définitions et mesures des variables du modèle.

\begin{tabular}{|c|c|c|c|c|c|}
\hline $\begin{array}{l}\text { Groupe de } \\
\text { variables }\end{array}$ & $\begin{array}{l}\text { Indicateurs de } \\
\text { mesure }\end{array}$ & Noms & Types & Modalités & $\begin{array}{l}\text { Auteurs de } \\
\text { reierence }\end{array}$ \\
\hline \multirow{4}{*}{ 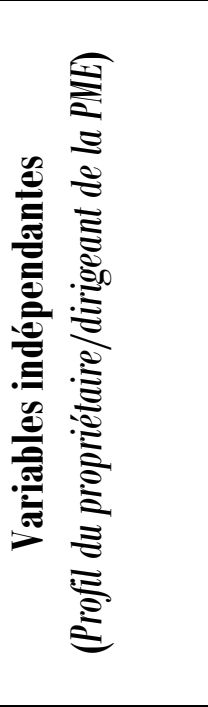 } & $\begin{array}{c}\text { Age du } \\
\text { propriétaire/dirigeant } \\
\text { de la PME }\end{array}$ & AP & $\begin{array}{l}\text { Polytomique } \\
\text { ordonnée }\end{array}$ & $\begin{array}{l}\mathbf{1} \mathrm{AP}<30 \text { ans } \\
2 \text { si } 30 \leq \mathrm{AP}<40 \text { ans } \\
3 \text { si } 40 \leq \mathrm{AP}<50 \text { ans } \\
4 \text { si } \mathrm{AP} \geq 50 \text { ans }\end{array}$ & $\begin{array}{l}\text { St-Pierre et al. (2011); } \\
\text { Hamadi et } \\
\text { Chapelier (2013) }\end{array}$ \\
\hline & $\begin{array}{l}\text { Niveau d'étude du } \\
\text { propriétaire }\end{array}$ & NEP & $\begin{array}{l}\text { Polytomique } \\
\text { ordonné }\end{array}$ & $\begin{array}{l}\mathbf{1} \text { si } \mathrm{NEP}=\text { Primaire } \\
2 \text { si NEP }=\text { Secondaire } 1^{\text {er }} \text { cycle } \\
\mathbf{3} \text { si } \mathrm{NEP}=\text { Secondaire 2e cycle } \\
\mathbf{4} \text { si NEP = Supérieur }\end{array}$ & $\begin{array}{l}\text { Mcclelland (1961); } \\
\text { Gartner (1980); } \\
\text { Phung (2009) }\end{array}$ \\
\hline & $\begin{array}{c}\text { Formation } \\
\text { particulière en } \\
\text { sciences de gestion } \\
\end{array}$ & FPSG & Dichotomique & $\begin{array}{l}\mathbf{1} \text { si 0ui } \\
\mathbf{0} \text { si Non }\end{array}$ & $\begin{array}{l}\text { McMahon et al. (1993); } \\
\text { St-Pierre (2004) }\end{array}$ \\
\hline & $\begin{array}{l}\text { Intensité du réseau } \\
\text { relationnel du } \\
\text { propriétaire }\end{array}$ & IRESP & Dichotomique & $\begin{array}{l}1 \text { si Elevée } \\
0 \text { si Faible }\end{array}$ & $\begin{array}{l}\text { Bourdieu }(1980) \text {, } \\
\text { Aydi }(2003) ; \\
\text { Feudjo et Tchankam } \\
(2012) ;\end{array}$ \\
\hline $\begin{array}{c}\text { Variable } \\
\text { dépendante }\end{array}$ & $\begin{array}{l}\text { Montant du crédit } \\
\text { reçu }\end{array}$ & CREDRECU & Dichotomique & $\begin{array}{l}\text { I si Elevé } \\
\mathbf{0} \text { si Faible }\end{array}$ & Djoutsa et al. (2018) \\
\hline
\end{tabular}

Source : auteur à partir de la littérature et de la méthodologie

\footnotetext{
${ }^{5}$ Les enquêtes par questionnaire ont eu lieu durant la période allant du $1^{\text {er }}$ Novembre 2018 au 31 Mars 2019 dans les villes de Yaoundé, Douala et Bafoussam qui représentent les villes les plus économiquement actives du Cameroun.
} 
Pour capter la variable Intensité du réseau relationnel du dirigeant (IRESP), plusieurs analyses préliminaires ont été effectuées. Ainsi, nous avons premièrement distingué entre la taille, l'importance et la qualité du réseau relationnel du propriétaire/dirigeant de l'entreprise en s'inspirant des travaux de Lin (1995), Nahapiet et Ghoshal (1998). Ensuite, en faisant recours à la méthodologie d'Aydi (2003) et de Feudjo et Tchankam (2012), le score global mesurant l'intensité du réseau relationnel du propriétaire/dirigeant (compris entre 16 et 32) a été calculé pour chaque individu de l'échantillon. La moyenne des notes extrêmes est de 24 . La modalité «forte » sera attribuée aux scores supérieurs à ce seuil (codée 1) et la modalité « faible » dan s le cas contraire (codée $\mathbf{0}$ ). Le tableau 2 suivant présente l'analyse préliminaire effectuée.

\section{Tableau 2: Mesure du réseau relationnel du dirigeant}

\begin{tabular}{|c|c|c|}
\hline \multirow[t]{2}{*}{$\begin{array}{l}\text { Nous avons distingué entre } \\
\text { la taille, l'importance et la } \\
\text { qualité du réseau relationnel. }\end{array}$} & \multicolumn{2}{|c|}{$\begin{array}{l}\text { Modalités et valeurs } \\
\text { numériques pour le calcul du } \\
\text { score }\end{array}$} \\
\hline & +1 & +2 \\
\hline \multicolumn{3}{|l|}{ Taille du réseau relationnel } \\
\hline $\begin{array}{r}\text { Existence des liens particuliers avec des hauts responsables } \\
\text { d'institutions bancaires }\end{array}$ & Non & Oui \\
\hline $\begin{array}{r}\text { Existence des liens particuliers avec des hauts responsables } \\
\text { de l'administration publique }\end{array}$ & Non & Oui \\
\hline $\begin{array}{r}\text { Existence des liens particuliers avec des hauts responsables } \\
\text { d'associations politiques }\end{array}$ & Non & Oui \\
\hline $\begin{array}{l}\text { Existence des liens particuliers avec des hauts responsables } \\
\text { d'institutions financières non bancaires }\end{array}$ & Non & Oui \\
\hline $\begin{array}{r}\text { Existence des liens particuliers avec des associations } \\
\text { culturelles ou religieuses }\end{array}$ & Non & Oui \\
\hline $\begin{array}{l}\text { Existence des liens particuliers avec des fournisseurs de } \\
\text { stocks de marchandises et autres approvisionnements }\end{array}$ & Non & Oui \\
\hline \multicolumn{3}{|c|}{ Importance du réseau relationnel } \\
\hline $\begin{array}{r}\text { Recours au réseau relationnel en cas de problèmes de } \\
\text { financement }\end{array}$ & Non & Oui \\
\hline $\begin{array}{r}\text { Degré d'importance accordé au réseau relationnel dans } \\
\text { l'accès au crédit }\end{array}$ & $\begin{array}{c}\text { Moins } \\
\text { important }\end{array}$ & Important \\
\hline $\begin{array}{c}\text { Degré d'importance accordé au réseau relationnel des } \\
\text { autres partenaires dans l'accès au crédit }\end{array}$ & $\begin{array}{c}\text { Sans } \\
\text { importance }\end{array}$ & Important \\
\hline Arguments privilégiés lors de la demande de crédit & $\begin{array}{l}\text { Performances } \\
\text { de } \\
\text { l'entreprise }\end{array}$ & $\begin{array}{l}\text { Liens particuliers } \\
\text { avec le banquier }\end{array}$ \\
\hline \multicolumn{3}{|c|}{ Qualité du réseau relationnel } \\
\hline $\begin{array}{r}\text { Qualité des relations avec des responsables d'institutions } \\
\text { bancaires }\end{array}$ & Faible & Elevée \\
\hline $\begin{array}{r}\text { Qualité des relations avec des associations culturelles ou } \\
\text { religieuses }\end{array}$ & Faible & Elevée \\
\hline $\begin{array}{r}\text { Qualité des relations avec de hauts responsables } \\
\text { d'associations politiques }\end{array}$ & Faible & Elevée \\
\hline $\begin{array}{c}\text { Qualité des relations avec des hauts responsables } \\
\text { d'institutions financières non bancaires }\end{array}$ & Faible & Elevée \\
\hline
\end{tabular}

Qualité des relations avec cercles et clubs d'amitié ou d'affaires 
Auteur à partir de nos analyses

Les données ont été analysées sur le logiciel SPSS à l'aide notamment des tableaux croisés dynamiques, du test du khi deux et de la régression logistique binaire.

\section{III- Résultats et discussions}

Dans la rubrique précédente, les variables d'études ont été présentées. Toutes ces variables ont été choisies à l'aide de la littérature existante et des échanges avec les propriétaires/dirigeants de PME du sous-secteur commerce/distribution. La présente rubrique s'attèle essentiellement à présenter les résultats d'un point de vue descriptif d'une part (3.1) et d'autre part, les résultats d'un point de vue économétrique (3.2).

\section{1- Analyse descriptive}

Le profil du propriétaire/dirigeant de la PME a été appréhendé à travers quatre variables à savoir l'âge, le niveau d'étude, la formation particulière en sciences de gestion et l'intensité du réseau relationnel. En ce qui concerne l'âge du propriétaire/dirigeant de l'entreprise, l'échantillon de l'étude (93 PME) a été subdivisé en quatre groupes ; le premier tient compte des promoteurs ayant moins de 30 ans, le second comprend les promoteurs dont l'âge oscille entre 30 et 40 ans, le troisième groupe concerne les promoteurs de PME dont l'âge se situe entre 41 et 50 ans et le quatrième groupe est constitué des promoteurs ayant plus de 50 ans. Le tableau 3 ci-dessous, met en relief les liens statistiques entre ces groupes de promoteurs et la probabilité d'accéder au montant du crédit sollicité.

Au regard des résultats présentés dans ce tableau, nous pouvons tirer quelques conclusions :

- après avoir scindé l'échantillon par rapport aux modalités de réponse que nous avons proposées aux promoteurs de PME, nous avons finalement obtenu 5 observations pour les propriétaires/dirigeants de PME âgés de

- moins de 30 ans, 29 observations pour ceux ayant un âge oscillant entre 30 et 40 ans, 39 observations pour ceux ayant un âge se situant entre 41 et 50 ans et 20 observations pour ceux ayant un âge supérieur à 50 ans ;

- pour ce qui est des promoteurs ayant moins de 30 ans, la probabilité élevée d'accéder au montant du crédit sollicité est observée dans $60 \%$ de cas et la probabilité faible d'accéder au montant du crédit sollicité est observée dans $40 \%$ de cas. Quant aux promoteurs âgés entre 30 et 40 ans, cette probabilité est élevée dans $72,4 \%$ de cas et faible dans $27,6 \%$ de cas. S'agissant des promoteurs âgés entre 41 et 50 ans, cette probabilité est élevée dans $74,4 \%$ de cas et faible dans $25,6 \%$ de cas ;

Tableau 3 : Age du promoteur de l'entreprise et montant du crédit reçu

\begin{tabular}{|c|c|c|c|c|c|}
\hline & & & \multicolumn{2}{|c|}{ Montant du crédit reçu } & \multirow[t]{2}{*}{ Total } \\
\hline & & & Faible & Elevé & \\
\hline \multirow{14}{*}{$\begin{array}{c}\text { Age du } \\
\text { promoteur de } \\
\text { l'entreprise }\end{array}$} & \multirow{4}{*}{$\begin{array}{r}\text { Moins de } \\
30 \text { ans }\end{array}$} & Effectif & 2 & 3 & 5 \\
\hline & & $\%$ (ligne) & $40,0 \%$ & $60,0 \%$ & $100,0 \%$ \\
\hline & & $\%$ (colonne) & $8,7 \%$ & $4,3 \%$ & $5,4 \%$ \\
\hline & & $\%($ total $)$ & $2,2 \%$ & $3,2 \%$ & $5,4 \%$ \\
\hline & \multirow{4}{*}{$\begin{array}{r}\text { Entre } 30 \text { et } \\
40 \text { ans }\end{array}$} & Effectif & 8 & 21 & 29 \\
\hline & & $\%$ (ligne) & $27,6 \%$ & $72,4 \%$ & $100,0 \%$ \\
\hline & & $\%$ (colonne) & $34,8 \%$ & $30,0 \%$ & $31,2 \%$ \\
\hline & & $\%($ total $)$ & $8,6 \%$ & $22,6 \%$ & $31,2 \%$ \\
\hline & \multirow{4}{*}{$\begin{array}{r}\text { Entre } 41 \text { et } \\
50 \text { ans }\end{array}$} & Effectif & 10 & 29 & 39 \\
\hline & & $\%$ (ligne) & $25,6 \%$ & $74,4 \%$ & $100,0 \%$ \\
\hline & & $\%$ (colonne) & $43,5 \%$ & $41,4 \%$ & $41,9 \%$ \\
\hline & & $\%($ total $)$ & $10,8 \%$ & $31,2 \%$ & $41,9 \%$ \\
\hline & \multirow[t]{2}{*}{ Plus de 50} & Effectif & 3 & 17 & 20 \\
\hline & & $\%$ (ligne) & $15,0 \%$ & $85,0 \%$ & $100,0 \%$ \\
\hline
\end{tabular}




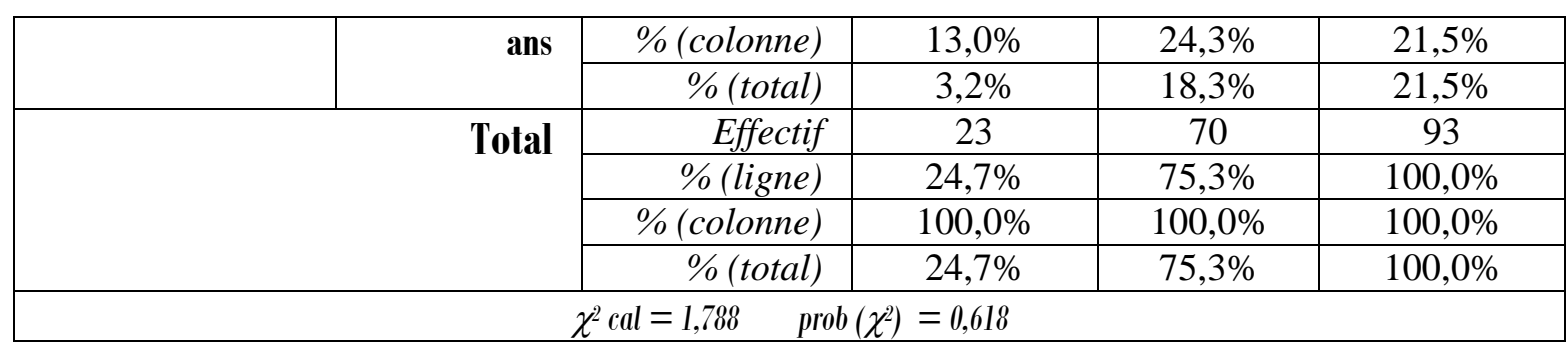

Source : auteur à partir des tests statistiques

- une comparaison relative de la probabilité de recevoir le montant du crédit sollicité entre les quatre catégories de PME montre que la modalité élevé est plus observée chez les promoteurs de PME âgés entre 41 et 50 ans $(41,4 \%)$, suivie de ceux dont l'âge se situe entre 30 et 40 ans (30\%).

Le test de Khi-deux montre qu'à priori, il n'existe aucun lien entre l'âge du promoteur de l'entreprise et la probabilité d'accéder au montant du crédit sollicité (signification asymptotique bilatérale $=0,618$ ).

Pour ce qui est du niveau d'étude du promoteur, l'échantillon a été subdivisé en quatre groupes; le premier comprend les promoteurs ayant un niveau d'étude primaire, le second tient compte des promoteurs ayant un niveau d'étude secondaire $1^{\text {er }}$ cycle, le troisième comporte les promoteurs ayant un niveau d'étude secondaire $2^{\mathrm{e}}$ cycle et le $4^{\mathrm{e}}$ comporte les promoteurs ayant un niveau d'étude supérieur. Le tableau 4 présente les liens statistiques existant entre ces différents niveaux d'étude et la probabilité d'accéder au montant du crédit sollicité.

Tableau 4 : Niveau d'étude du promoteur et montant du crédit reçu

\begin{tabular}{|c|c|c|c|c|c|}
\hline & & & \multicolumn{2}{|c|}{ Montant du crédit reçu } & \multirow[t]{2}{*}{ Total } \\
\hline & & & Faible & Elevé & \\
\hline \multirow{16}{*}{$\begin{array}{c}\text { Niveau } \\
\text { d'étude du } \\
\text { promoteur }\end{array}$} & \multirow[t]{4}{*}{ Primaire } & Effectif & 2 & 11 & 13 \\
\hline & & $\%$ (ligne) & $15,4 \%$ & $84,6 \%$ & $100,0 \%$ \\
\hline & & $\%$ (colonne) & $8,7 \%$ & $15,7 \%$ & $14,0 \%$ \\
\hline & & $\%($ total $)$ & $2,2 \%$ & $11,8 \%$ & $14,0 \%$ \\
\hline & \multirow{4}{*}{$\begin{array}{r}\text { Secondaire } \\
\text { premier cycle }\end{array}$} & Effectif & 9 & 25 & 34 \\
\hline & & $\%$ (ligne) & $26,5 \%$ & $73,5 \%$ & $100,0 \%$ \\
\hline & & $\%$ (colonne) & $39,1 \%$ & $35,7 \%$ & $36,6 \%$ \\
\hline & & $\%($ total $)$ & $9,7 \%$ & $26,9 \%$ & $36,6 \%$ \\
\hline & \multirow{4}{*}{$\begin{array}{r}\text { Secondaire } \\
\text { deuxième cycle }\end{array}$} & Effectif & 7 & 23 & 30 \\
\hline & & $\%$ (ligne) & $23,3 \%$ & $76,7 \%$ & $100,0 \%$ \\
\hline & & $\%$ (colonne) & $30,4 \%$ & $32,9 \%$ & $32,3 \%$ \\
\hline & & $\%($ total $)$ & $7,5 \%$ & $24,7 \%$ & $32,3 \%$ \\
\hline & \multirow[t]{4}{*}{ supérieur } & Effectif & 5 & 11 & 16 \\
\hline & & $\%$ (ligne) & $31,3 \%$ & $68,8 \%$ & $100,0 \%$ \\
\hline & & $\%$ (colonne) & $21,7 \%$ & $15,7 \%$ & $17,2 \%$ \\
\hline & & $\%($ total $)$ & $5,4 \%$ & $11,8 \%$ & $17,2 \%$ \\
\hline \multirow{4}{*}{\multicolumn{2}{|c|}{ Total }} & Effectif & 23 & 70 & 93 \\
\hline & & $\%$ (ligne) & $24,7 \%$ & $75,3 \%$ & $100,0 \%$ \\
\hline & & $\%$ (colonne) & $100,0 \%$ & $100,0 \%$ & $100,0 \%$ \\
\hline & & $\%$ du total & $24,7 \%$ & $75,3 \%$ & $100,0 \%$ \\
\hline \multicolumn{3}{|c|}{$\chi^{2} c a l=4,062$} & $\left.\chi^{2}\right)=0$ & & \\
\hline
\end{tabular}

Source : auteur à partir des tests statistiques

En observant ce tableau, on remarque que :

après avoir scindé cet échantillon par rapport aux modalités de réponse que nous avons proposées aux propriétaires/dirigeants de PME, nous avons en fin de compte eu 13 observations pour ceux ayant un niveau d'étude primaire, 34 observations pour ceux ayant un niveau d'étude secondaire $1^{\text {er }}$ cycle, 30 
observations pour ceux ayant un niveau secondaire $2^{\mathrm{e}}$ cycle et 16 observations pour les promoteurs ayant un niveau d'étude supérieur ;

$>$ la probabilité de recevoir un montant de crédit élevé est observée dans plus de $68 \%$ de cas des propriétaires/dirigeants de PME ayant au moins un niveau d'étude secondaire $2^{\mathrm{e}}$ cycle ;

$>$ une comparaison relative des niveaux de probabilité d'accéder au montant du crédit sollicité montre que la modalité «élevé » est plus observée chez les promoteurs de PME ayant un niveau d'étude secondaire premier cycle $(35,7 \%)$.

Le test de Khi-deux montre qu'à priori, il existe une relation entre le niveau d'étude du promoteur de l'entreprise et la probabilité d'accéder au montant du crédit sollicité (signification asymptotique bilatérale = 0,076).

Pour ce qui est de la formation particulière en sciences de gestion, l'échantillon a été subdivisé en deux groupes ; le premier comprend les promoteurs ayant reçu une formation particulière en sciences de gestion et le second tient compte des promoteurs n'ayant pas reçu de formation particulière en sciences de gestion. Le tableau 5 présente les liens statistiques existant entre ces deux groupes et la probabilité d'accès au montant du crédit sollicité.

À la lecture de ce tableau, on relève que :

$>$ après avoir scindé cet échantillon par rapport aux modalités de réponse que nous avons proposées aux dirigeants, nous avons en fin de compte eu 27 observations pour les promoteurs ayant reçu une formation particulière en sciences de gestion contre 66 qui ne l'ont pas reçu ;

$>$ parmi les propriétaires/dirigeants de PME du sous-secteur commerce/distribution ayant reçu une formation particulière en sciences de gestion, la probabilité élevée d'accéder au montant du crédit sollicité est plus observée (70,4\%) que la probabilité faible d'accéder au montant du crédit sollicité (29,6\%).

Le test de Khi-deux montre qu'à priori, il existe un lien entre la formation particulière en sciences de gestion et la probabilité d'accéder au montant du crédit sollicité (signification asymptotique bilatérale $=0,044$ ).

Tableau 5 : Formation particulière en sciences de gestion et montant du crédit reçu

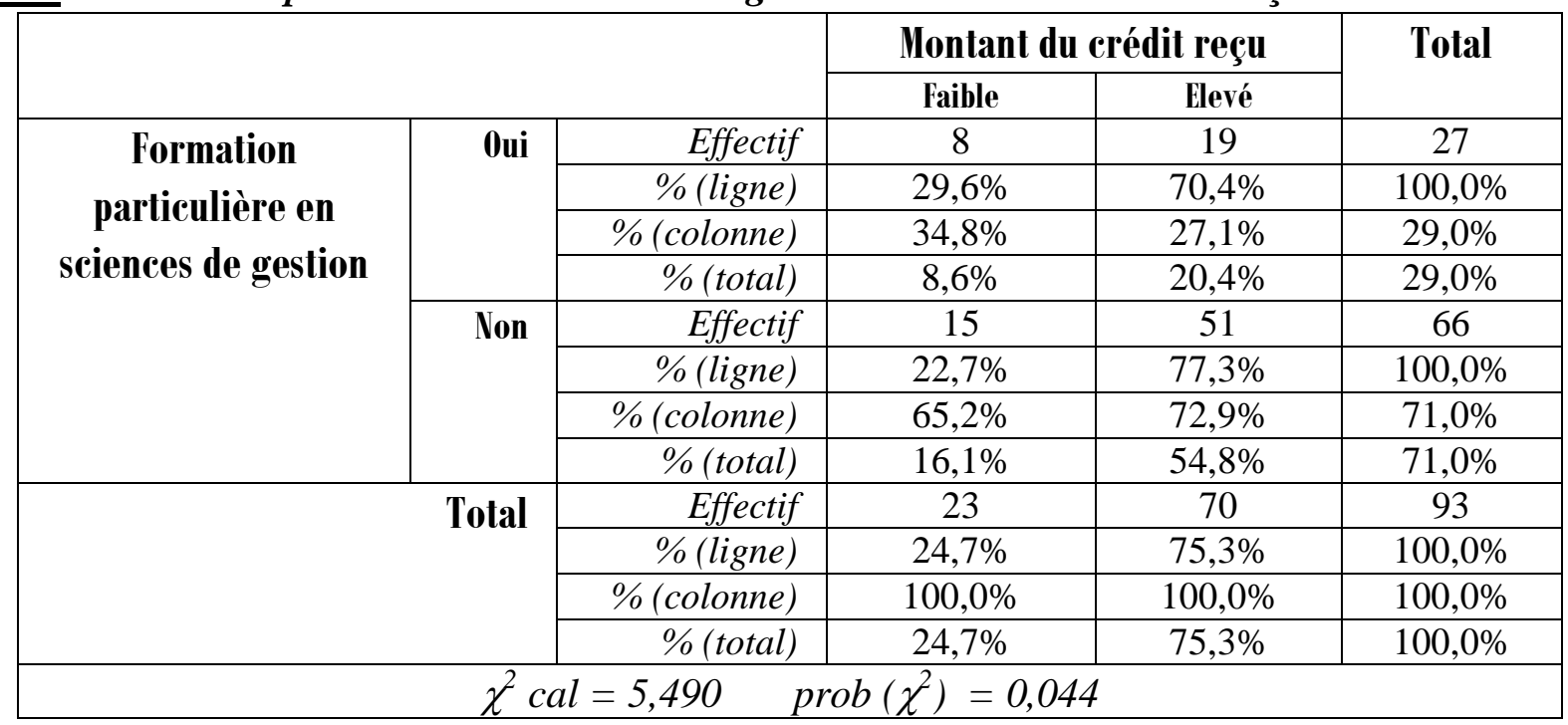

Source : auteur à partir des tests statistiques

En ce qui concerne l'intensité du réseau relationnel du propriétaire/dirigeant de la PME, l'échantillon a été subdivisé en deux compartiments ; le premier compartiment prend en compte les promoteurs de PME $\mathrm{du}$ sous-secteur commerce/distribution ayant une intensité de réseau relationnel faible et le deuxième compartiment tient compte des promoteurs de PME ayant une intensité de réseau relationnel élevée. Le 
tableau 6 présente les relations statistiques entre ces deux groupes de promoteurs et la probabilité d'accéder au montant du crédit sollicité.

Tableau 6 : Intensité du réseau relationnel du promoteur et montant du crédit reçu

\begin{tabular}{|c|c|c|c|c|c|}
\hline & \multicolumn{2}{|c|}{ Montant du crédit reçu } & \multirow[b]{2}{*}{ Total } \\
\hline & & & Faible & Elevé & \\
\hline \multirow{8}{*}{$\begin{array}{l}\text { Intensité du } \\
\text { réseau } \\
\text { relationnel } \\
\text { du } \\
\text { promoteur }\end{array}$} & \multirow[t]{4}{*}{ Faible } & Effectif & 4 & 9 & 13 \\
\hline & & $\%$ (ligne) & $30,8 \%$ & $69,2 \%$ & $100,0 \%$ \\
\hline & & $\%$ (colonne) & $17,4 \%$ & $12,9 \%$ & $14,0 \%$ \\
\hline & & $\%($ total) & $4,3 \%$ & $9,7 \%$ & $14,0 \%$ \\
\hline & \multirow[t]{4}{*}{ Elevée } & Effectif & 19 & 61 & 80 \\
\hline & & $\%$ (ligne) & $23,8 \%$ & $76,3 \%$ & $100,0 \%$ \\
\hline & & $\%$ (colonne) & $82,6 \%$ & $87,1 \%$ & $86,0 \%$ \\
\hline & & $\%($ total $)$ & $20,4 \%$ & $65,6 \%$ & $86,0 \%$ \\
\hline \multirow{4}{*}{\multicolumn{2}{|c|}{ Total }} & Effectif & 23 & 70 & 93 \\
\hline & & $\%$ (ligne) & $24,7 \%$ & $75,3 \%$ & $100,0 \%$ \\
\hline & & $\%$ (colonne) & $100,0 \%$ & $100,0 \%$ & $100,0 \%$ \\
\hline & & $\%($ total $)$ & $24,7 \%$ & $75,3 \%$ & $100,0 \%$ \\
\hline \multicolumn{6}{|c|}{$\chi^{2} c a l=4,296$} \\
\hline
\end{tabular}

Source : auteur à partir des tests statistiques

Au regard de ce tableau, nous pouvons tirer quelques enseignements :

- nous avons finalement eu 13 observations pour les propriétaires/dirigeants de PME ayant une intensité de réseau relationnel faible contre 80 observations pour ceux ayant une intensité de réseau relationnel élevée ;

- $76,3 \%$ de promoteurs ayant une intensité de réseau relationnel élevée ont une probabilité élevée d'accéder au montant du crédit bancaire sollicité.

- d'une comparaison relative, il ressort que la probabilité de recevoir un montant de crédit élevé est plus observée chez les promoteurs de PME ayant une intensité de réseau relationnel élevée $(87,1 \%)$ par rapport aux propriétaires/dirigeants de PME ayant une intensité de réseau relationnel faible $(12,9 \%)$.

Le test de Khi-deux montre qu'à priori, il existe un lien entre l'intensité du réseau relationnel du propriétaire/dirigeant de la PME et la probabilité d'accéder au montant du crédit sollicité (signification asymptotique bilatérale $=0,066$ ).

\section{2- Résultats de l'analyse explicative : régression logistique}

Toutes les variables retenues pour capter le profil du propriétaire/dirigeant de la PME se sont avérées significatives comme le montre le tableau 7. L'exploitation de ce tableau permet de tirer quelques enseignements.

Tableau 7 : Profil du propriétaire/dirigeant de la PME et probabilité d'accès au montant du crédit sollicité : une estimation logit.

\begin{tabular}{|r|l|l|}
\hline \multirow{2}{*}{ Modalités } & \multicolumn{1}{|c|}{ Modèle } \\
\cline { 2 - 3 } & Coef. & Odds-R \\
\hline \multicolumn{3}{|c|}{$\mathbf{A P}$} \\
\hline Entre 30 et 40 ans & $\mathbf{1 , 2 2 8}$ & 3,414 \\
& $(0,313)$ & \\
\hline Entre 41 et 50 ans & $\mathbf{0 , 5 6 9} *$ & 1,766 \\
& $(0,063)$ & \\
\hline Plus de 50 ans & $\mathbf{0 , 6 1 6} * *$ & 1,851 \\
& $(0,025)$ & \\
\hline \multicolumn{3}{|c}{$\mathbf{N E P}$} \\
\hline
\end{tabular}




\begin{tabular}{|c|c|c|}
\hline Secondaire $1^{\text {er }}$ cycle & $\begin{array}{r}-\mathbf{0 , 3 4 0} \\
(0,782)\end{array}$ & 0,712 \\
\hline Secondaire $2^{\text {nd }}$ Cycle & $\begin{array}{l}\mathbf{0 , 0 7 3} * \\
(0,052)\end{array}$ & 1,076 \\
\hline Supérieur & $\begin{array}{c}\mathbf{0 , 3 3 3} \\
(0,697)\end{array}$ & 1,396 \\
\hline FPSG (Non) & $\begin{array}{r}-\mathbf{0 , 2 3 0} * \\
(0,089) \\
\end{array}$ & 0,794 \\
\hline IRESP (Elevée) & $\begin{array}{l}\mathbf{0 , 2 4 5} * * * \\
(0,009)\end{array}$ & 1,278 \\
\hline Constante & $\begin{array}{c}\mathbf{1 , 6 2 8} \\
(0,145)\end{array}$ & 5,096 \\
\hline $\begin{array}{l}\text { Khi-deux : } \mathbf{7 , 6 5 2} * \\
\mathrm{R}^{2} \text { de Nagelkerke : } \mathbf{0 , 1 4}\end{array}$ & \multicolumn{2}{|c|}{$\begin{array}{l}\mathrm{R}^{2} \text { de Cox et Snell : } \mathbf{0 , 1 2 8} \\
\text { Pourcentage global : } \mathbf{7 5 , 3} \%\end{array}$} \\
\hline
\end{tabular}

$\underline{\text { Notes }}$ : Les valeurs entre parenthèses représentent les p-value et les astérisques indiquent les niveaux de significativité des coefficients : ***,** et $*$, respectivement significatifs à $1 \%, 5 \%$ et $10 \%$.

Taille de l'échantillon : 93 PME

\section{Source : Construction de l'auteur à partir des estimations.}

Il existe un lien positif et significatif entre l'âge du propriétaire/dirigeant de la PME du sous-secteur commerce/distribution et la probabilité d'accéder au montant du crédit sollicité. En effet, les propriétaires/dirigeants de PME âgés de plus de 40 ans ont une probabilité élevée de recevoir le montant total du crédit sollicité comparativement à ceux qui ont un âge inférieur à 40 ans. Au fur et à mesure qu'un promoteur de PME prend de l'âge, il gagne en expérience. Ce sens positif peut s'expliquer par l'intensification de la relation de confiance au fil du temps entre les promoteurs de PME et les banques. D'ailleurs, les travaux de Chebil et Levy Tadjine (2010) qui démontrent que l'expérience du dirigeant est un facteur déterminant de la décision d'octroi de crédit ; les jeunes sont des emprunteurs à très haut risque et que cette catégorie de dirigeant a un accès plus difficile au crédit. Ce résultat est conforme à nos attentes et épouse ceux d'Edwards et Fisher (1994), Berger et Udell (1998) qui avaient trouvé que l'âge de l'entreprise influence positivement et fortement sur sa probabilité d'obtention du crédit. Ce résultat est également conforme à celui trouvé par Phung (2009) au Vietnam ainsi qu'à celui trouvé par Djoutsa et al (2018) en contexte Camerounais à travers l'étude de 262 dossiers de demande de crédit auprès d'une banque. Toutefois, ce résultat est contraire à celui de Colot et Croquet (2007a) en contexte belge selon lequel, il existe une relation négative entre l'âge du propriétaire/dirigeant et la probabilité d'accès au crédit bancaire. Ce résultat est aussi contraire à celui trouvé par Ngongang et Ahmadou (2018) qui, partant d'un échantillon de 59 PME du secteur privé camerounais, aboutissent à la conclusion selon laquelle l'âge du propriétaire/dirigeant n'a aucune influence significative sur la probabilité d'accès au crédit bancaire des entreprises. Par rapport, à cette variable, l'hypothèse $\mathrm{H}_{1}$ selon laquelle l'âge du propriétaire/dirigeant de la $\mathrm{PME} / \mathrm{CD}$ influence positivement l'accès au montant du crédit bancaire sollicité est validée.

Concernant le niveau d'étude du propriétaire/dirigeant de la PME, les résultats montrent qu'il existe une relation positive et significative entre cette variable la probabilité de recevoir le montant du crédit sollicité. En effet, les promoteurs de PME ayant un niveau d'étude secondaire deuxième cycle ont une probabilité élevée d'accéder à la totalité du crédit sollicité par rapport à leurs homologues qui ont un niveau d'étude primaire. Ce résultat est conforme à nos attentes et rejoint celui obtenu par Moumou (2016) selon lequel les dirigeants ayant une formation de niveau supérieur accèdent plus facilement au crédit bancaire car les banquiers considèrent que ces derniers disposent d'un fort capital humain, source de développement des compétences managériales. Toutefois, ce résultat est contraire à celui de Le et al. (2006) qui trouvent que l'impact du niveau d'étude sur l'accès au crédit n'est pas appuyé empiriquement. Par rapport, à cette 
variable, l'hypothèse $\mathrm{H}_{2}$ selon laquelle Moins le niveau d'étude du propriétaire/dirigeant de la PME/CD est élevé, faible est sa probabilité d'accéder au montant du crédit bancaire sollicité est validée.

Les résultats montrent également un impact négatif et significatif entre le fait que le propriétaire/dirigeant de la PME ait reçu une formation particulière en sciences de gestion et la probabilité d'accéder à la totalité du crédit sollicité. Ce résultat est contraire à nos attentes et à celui de Pigé (2002) qui démontre que plus le dirigeant dispose d'un fort capital humain, plus il a des chances d'accéder au montant du crédit sollicité. Ce résultat est également en contradiction avec ceux de McMahon et al. (1993) et St-pierre (2004) qui pensent que le dirigeant ayant une formation en sciences de gestion est capable d'opérer des contrôles financiers réduisant ainsi le risque de faillite de l'entreprise et par conséquent, a plus de chances d'accéder au montant du crédit sollicité. Ce résultat est également contraire à celui de Ngongang et Ahmadou (2018) en contexte camerounais selon lequel, il n'existe aucune différence significative entre le niveau d'endettement bancaire des PME dont les dirigeants ont reçu une formation en sciences de gestion de celles dont les dirigeants ne sont pas formés en sciences de gestion. Par rapport, à cette variable, l'hypothèse $\mathrm{H}_{3}$ selon laquelle le fait pour le propriétaire/dirigeant de la $\mathrm{PME} / \mathrm{CD}$ d'avoir suivi une formation particulière en sciences de gestion augmente significativement sa probabilité d'accéder au montant du crédit sollicitée n'est pas validée.

En ce qui concerne l'intensité du réseau relationnel du propriétaire/dirigeant qui est en fait l'indicateur du dynamisme du réseau social du dirigeant, les résultats montrent que cette variable influence positivement et significativement la probabilité d'accéder à la totalité du crédit sollicité. En fait, les promoteurs de PME ayant une intensité de réseau relationnel élevée accèdent davantage au crédit par rapport à ceux qui ont une intensité de réseau relationnel faible. Ce résultat est conforme à nos attentes et corrobore la thèse développée en l'espèce par Aydi (2003) et Baudasse et Montalieu (2005) sur le rôle du capital social, comme un attribut de l'individu dans un contexte social qui peut être acquis à travers une action délibérée et transformé en gains économiques conventionnels selon la nature des connexions et des réseaux disponibles (Bourdieu, 1986). Il est également conforme à ceux de Fukuyama (1995), Putnam (1995) qui avaient montré que les réseaux associatifs procurent à leurs membres les avantages financiers parmi lesquels l'accès au crédit. Il est également conforme à la pensée de Labazee (1995) qui avait déjà trouvé qu'en Afrique, le réseau social constitue la principale source, voire exclusive du financement des activités du petit commerce. Cette position est d'ailleurs confortée par les travaux récents de Feudjo et Tchankam (2012) qui expliquent l'accès des entreprises surendettées au crédit bancaire par leur intensité de réseau relationnel ainsi que celui de Wamba et Niyonsaba (2014) qui trouvait déjà que le capital social a un effet positif et significatif sur l'accès au crédit bancaire des PME quel soit le terme dans un environnement caractérisé par de fortes asymétries d'informations. Dans la même veine, se situent également les résultats des travaux de Kenfack (2016). De plus, ce résultat est conforme à ceux trouvés par Diamond (1991), Labazee (1995), Petersen et Rajan (1997), Berger et Udell (2002), Aydi (2003). Ces résultats épousent les postulats de la théorie du réseau social. Par rapport, à cette variable, l'hypothèse $\mathrm{H}_{4}$ selon laquelle le réseau relationnel du propriétaire/dirigeant de la $\mathrm{PME} / \mathrm{CD}$ impacte positivement sur la probabilité d'accéder au montant du crédit bancaire sollicité est validée.

\section{Conclusion}

Les PME constituent l'essentiel des entreprises aussi bien dans les pays développés qu'émergents. Elles sont à la base de la croissance économique dans tous les secteurs d'activité. Au-delà de leur capacité à créer des emplois et de la richesse, elles sont confrontées à des difficultés dès qu'il s'agit d'obtenir des financements d'origine bancaire. La présente étude a eu pour ambition de déterminer l'impact du profil du propriétaire/dirigeant de la $\mathrm{PME} / \mathrm{CD}$ sur l'accès au crédit bancaire au Cameroun. Nous avons à cet effet, intégré comme profil du propriétaire/dirigeant, son âge, son niveau d'étude, sa formation particulière en Sciences de gestion et son réseau relationnel. 
L'exploitation d'un questionnaire nous a permis de collecter les informations auprès de 93 propriétaires/dirigeants de $\mathrm{PME} / \mathrm{CD}$ ayant déjà bénéficié du crédit bancaire par le passé. Les résultats de l'estimation par régression logistique binaire mettent en exergue, un effet significatif du profil du propriétaire/dirigeant sur la probabilité d'accéder au montant du crédit sollicité au Cameroun. Cette probabilité est d'autant plus élevée lorsque le propriétaire/dirigeant a 40 ans et plus, un niveau d'étude secondaire deuxième cycle et une intensité de réseau relationnel élevée.

La faible taille de l'échantillon et le fait d'avoir mené notre enquête dans 3 villes du Cameroun limitent la généralisation des résultats. Les études ultérieures vont s'intéresser à l'élargissement de la taille de l'échantillon et à la prise en compte de toutes les villes représentants les chefs-lieux des 10 régions que compte le Cameroun

\section{References}

[1] Ang J.S (1992), «On the theory of finance for private held firms », The Journal of Small Business Finance, Vol. 1, pp. 185-203

[2] Aydi G. (2003), « Capital social entrepreneurial, performance de l'entreprise et accès aux ressources externes », XII ${ }^{\mathrm{e}}$ Conférence de l'Association internationale de management stratégique, Carthage, Tunisie, 3-6 juin.

[3] Baudasse T. et Montalieu T. (2005), « Le capital social : un concept utile pour la finance et le développement durable », IX Journées scientifiques de l'Agence universitaire de la Francophonie, Cluj-Napoca, Roumanie, juin.

[4] Belletante B. et Levratto N. (1995), «Finance et PME : quels champs pour quels enjeux ? », Revue internationale PME, Vol. 8, $\mathrm{n}^{\circ}$ 3-4, pp. 5-42.

[5] Berger A. N. et Udell G. F. (1988), «The Economics of Small Business Finance: The Roles of Private Equity and Debt Markets in the Financial Growth Cycle », Journal of Banking and Finance, Vol. 22, pp. 613-673.

[6] Boukar H. et Tsapi V. (2011), «Impact du profil socioculturel du dirigeant sur la croissance des micros et petites entreprises camerounaises », Revue des Sciences de Gestion, n77, $\quad$ pp. 1933.

[7] Bourdieu P. (1980), «Le capital social : notes provisoires », Actes de la recherche en sciences sociales, Vol. 3, 2-3.

[8] Bourdieu P., Boltanski L. et De Saint-Martin M. (1973), «Les stratégies de reconversion », Social Science Information, Vol. 12, n5, pp. 61-113.

[9] Chebil A. et Levy-Tadjine T. (2010), « Décision d'octroi de crédit bancaire aux PME et diversité des critères d'évaluation par le banquier », Document de Travail Non publié.

[10] Coleman J-S (1990), «Foundation of Social Theory », Cambridge: MA, Havard University Press.

[11] Colot O. et Croquet M. (2007a), « La contribution de variables propres aux PME et à leur dirigeant dans l'explication de la structure financière des PME », La Revue des Sciences de Gestion, vol. 6, $\mathrm{n}^{\circ}$ 228, pp. 61-72.

[12] Cooper A. (1993), « Challenges in Predicting New Firm Performance », Journal of Business Venturing, Vol. 8, pp. 243.

[13] Coupal, M. (1994), «La PME, copie conforme de son fondateur », Revue Organisation, Vol 3, n¹, p.39-44.

[14] D'Amboise G. (1997), « Quelle gestion stratégique pour la PME », Québec-Canada, Presses Inter Universitaire.

[15] Djoutsa W. L., Sahut J. M. et Teulon F. (2018), « L'importance des dimensions temporelles de la relation banque-PME sur la décision d'octroi de crédit bancaire dans un contexte d'asymétrie d'information », Gestion 2000, Vol. 35, pp. 77-95. 
[16] Fathi E. T. et Gailly B. (2003), «La structure financière des PME de la haute technologie », XII ${ }^{\mathrm{e}}$ Conférence de l'Association internationale de management stratégique, Tunis, 3 et 6 juin, pp. 22-35.

[17] Feudjo J. R. et Tchankam J. P. (2012), « Les déterminants de la structure financière: comment expliquer le paradoxe de l'insolvabilité et de l'endettement des PMI au Cameroun ? », Revue Internationale PME, Vol. 25, $\mathrm{n}^{\circ}$ 2, pp. 100-128.

[18] Gartner W.B. (1988), "Who is an Entrepreneur? Is the Wrong Question », American Journal of Small Business, Vol. 12, n 4 pp. 11-31.

[19] Granovetter M. (1973), « The Strength of Weak Ties », American Journal of Economics and Sociology, Vol. 78, n³, pp. 1360-1380.

[20] Filion, L.J. (1991), Vision et relations : clefs du succès de l'entrepreneur, Les éditions de l'entrepreneur, Montréal, $272 \mathrm{p}$.

[21] INS (2018), «Recensement Général des Entreprises 2016, rapport préliminaire des principaux résultats », Janvier, $40 \mathrm{p}$.

[22] Janssen F. et Wtterwulghe R. (1998), «L'influence de l'interpénétration du dirigeant et de son entreprise sur l'endettement bancaire des PME: Etat de la question », Université Catholique de Louvain : Centre Européen de la PME, Institut d'Administration et de Gestion.

[23] Jensen M. C. et Meckling W. (1976), « Theory of the Firm Managerial Behaviour, Agency Costs and Ownership Structure », Journal of Financial Economics, Vol. 3, Issue 4, October, pp. 305- 360.

[24] Julien, P.A. (1990), "Vers une typologie multicritères des PME", Revue Internationale PME, Vol 3, n 3-4, p.411-425.

[25] Mcclelland D. (1961), The Achieving Society, Van Nostrand, Princeton NJ, 1961.

[26] Modigliani F. et Miller M. (1958), «The Cost of Capital, Corporate Finance and the Theory of Investment », American Economic Review, Vol. 58, n 3, pp. 261-297.

[27] Nahapiet J. et Ghoshal S. (1998), « Social Capital, Intellectual Capital, and the Organizational Advantage », Academy of Management Review, Vol. 23, n², pp. 242-266.

[28] Myers, S. \& Majluf, N. (1984), « Corporate Financing and Investment Decision When Firm Have Information That Investor Do Not Have », Journal of Financial Economics, Vol. 13, pp. 187-221.

[29] Ndjanyou L. (2001) «Risque, Incertitude et Financement bancaire de la PME camerounaise : l'exigence d'une analyse spécifique du risqué », Document of Center for Economic Research on Africa (CERAF), Montclair State University, Upper Montclair, New Jersey 07043.

[30] Nkakleu, R. (2003), " L’identité organisationnelle et création du capital social : la tontine d'entreprise comme facteur déclenchant dans le contexte africain », Actes des XVIe Journées nationales de l'IAE, Paris, 10-12 septembre.

[31] Ngantchou A. (2010), Contenu informatif des normes comptables, profil moral des préparateurs des chiffres et essai d'explication de la discrimination des PME sur le marché du financement bancaire : une étude empirique sur les données camerounaises, Thèse de doctorat en Sciences de gestion, Université de Douala.

[32] Ngongang D. et Ahmadou B. (2018), «Impact du profil du dirigeant sur le niveau d'endettement bancaire des PME camerounaises », Journal of Academic Finance, Vol. 9, n¹, pp. 1-16.

[33] Putnam R.D. (1995), « Bowling Alone: America's Declining Social Capital », Journal of Democracy, Vol. 6, n¹, pp. 65-78.

[34] Ross .A. (1977), «The Definition of Financial Structure: The Incentive Signaling Approach », Journal of Economics, Printemps, pp. 23-40.

[35] Saporta B. (1994), «La création d'entreprises : enjeux et perspectives», Revue Française de Gestion, n¹01, Novembre-Décembre. 
[36] Schwarz S. (2011), « Les difficultés des Entrepreneurs pour accéder au crédit en République Démocratique du Congo », KfW Bankengruppe, Département de la Communication Palmengartenstr, Germany.

[37] Stewart et Myers (2001), «Capital Structure », The Journal of Economic Perspectives, Vol. 15, $\mathrm{n}^{\circ} 2$, pp. 81.

[38] St-pierre J. (2004), « La gestion du risque: comment améliorer le financement des PME et faciliter leur développement », Presses de l'Université du Québec, Canada.

[39] Um-Ngouem M.T. et Ekonne E.A. (2013), « Facteurs d'éligibilité des Très Petites Entreprises (TPE) au crédit des Etablissements de Microfinance (EMF) au Cameroun : une étude exploratoire », Communication aux $\mathrm{V}^{\text {ème }}$ journées internationales de la micro finance, Université d'Orléans, 11, 12 et 13 septembre.

[40] Uzzi B. (1999), «Embeddedness in the Making of Financial Capital: How Social Relations and Networks Beneft Firms Seeking Financing », American Sociological Review, Vol. 64, n4, pp. 481 505.

[41] Wamba H. et Tchamanbe-Djine L. (2002), « Information financière et politique d'offre de crédit bancaire aux PME : cas du Cameroun », Revue Internationale PME, $2^{\mathrm{e}}$ trimestre, Vol. 15, $\mathrm{n}^{\circ} 1$, pp. 87-145.

[42] Wanda R. (2007), «Risques, comportements bancaires et déterminants de la surliquidité », La Revue des Sciences de Gestion, Direction et Gestion des entreprises, n²28, pp. 93-102.

[43] Watts, R. L. et Zimmerman, J. L. (1990), «Positive Accounting Theory: A Ten Year Perspective », The Accounting Review, January, Vol. 65 n ${ }^{\circ} 1, \quad$ pp. 131-156. 